\title{
Third molar surgery and postoperative pain relief
}

\author{
Changes in life quality following third molar surgery - the immediate postoperative period. \\ C. McGrath, M. B. Comfort, E. C. M. Lo and Y. Luo Br Dent J 2003; 194: 265-268
}

\section{Objectives}

This study describes patients' perceptions of changes in oral health related quality of life (OHQOL) in the early postoperative period following third molar surgery.

\section{Methods}

One hundred patients were enrolled in a prospective cohort study of the surgical removal of lower third molars under local anaesthetic. Two specific oral health related quality of life measures, OHIP-14 and OHQoLUK ${ }^{\odot}$ were administered to the study group prior to surgery. Standardized surgical and analgesic protocols were followed. Patients kept a diary of changes in life quality each postoperative day (POD) for 7 days, completing both OHIP-14 and OHQoLUK ${ }^{\odot}$ daily.

\section{Results}

Both oral health related quality of life measures identified a significant deterioration in quality of life on POD1 $(\mathrm{P}<0.01)$ and this remained evident on POD2 $(\mathrm{P}<0.01)$, P0D3 $(\mathrm{P}<0.01)$, P0D4 $(\mathrm{P}<0.01)$ and POD5 $(\mathrm{P}<0.05)$. By P0D6 and P0D7 there was no significant difference in quality of life compared with preoperative status $(\mathrm{P}>0.05)$. Deterioration in life quality over the study period was associated with postoperative clinical findings $(\mathrm{P}<0.05)$ : swelling and trismus.

\section{Conclusion}

The study concludes that there is a significant deterioration in oral health related quality of life in the immediate postoperative period following third molar surgery; particularly during the first five days. This is associated with postoperative clinical findings. This has implication for patients deciding on third molar surgery and informed consent.

\section{IN BRIEF}

- Almost all adults face the decision as to whether to have their third molars removed or not.

- Patients who were part of a prospective cohort study kept a diary of changes in their life quality each day for 7 days following third molar surgery and reported immense disturbances to their daily living

- Greater understanding of the outcomes of dental treatment from patients' perspectives can help to inform patients' treatment decision making processes and facilitate informed consent.

\section{COMMENT}

This paper sets out to assess changes in quality of life during the first week following removal of third molars. Given the number of wisdom teeth removed annually, this is still a relatively under investigated area of clinical practice. ${ }^{1}$ A number of oral health specific quality of life measures (questionnaires) have been developed including those specific for third molar removal. ${ }^{2}$ These may become important tools for assessing quality, effectiveness and efficiency of treatment.

One hundred patients were recruited who were to have one lower wisdom tooth removed under local anaesthesia. The patients' quality of life was assessed pre-operatively and then daily by the patient using the oral health impact profile (OHIP-14) and the UK oral health related quality of life measure (OHOLLUK). Patients were asked to complete a logbook each day until reviewed on day 7. Poor quality of life was reflected in a high OHIP score and a low OHQoLUK score.

Of the 100 patients recruited, 93 completed the study. The mean and medium ages were 26 and 24 respectively which mirrors the usual group requiring third molar removal. In the immediate postoperative period, there was a significant decrease in quality of life measured by both questionnaires. By day 7 this had returned to preoperatively values. The straightforward removal of an upper third molar did not appear to influence quality of life scores. Given that there has been a great deal of debate over specific clinical indications for removal of third molars, it would have been interesting to see if there were any differences in quality of life scores for those patients that satisfied guidelines for removal compared with those that did not. Also to assess the influence of type of impaction, bone removal, and length of operation on quality of life scores. The slight improvement in quality of life at day 7 compared with day 0 , suggests that some patients' quality of life was affected by third molar problems.

A previous study found that 1 in 2 patients still experienced pain one week postoperatively despite analgesic therapy. ${ }^{1}$ This paper provides further evidence of the urgent need to develop better pain control for patients requiring third molar removal.

Where there was no swelling and no trismus, there was often no change in quality of life but this was not always so; about a third reported a reduced quality of life without swelling or trismus. What other factors were responsible for this decrease in quality of life?

The little difference between day 0 and day 7 scores suggests that such influences are short lived. However, deterioration in quality of life was experienced across a broad range of domains (physical, social and psychological) and thus has relevance for counselling and obtaining informed consent for such procedures. Those involved in the construction of patient information leaflets should ensure that quality of life issues are included beyond the traditional ones of pain, swelling, bruising, trismus and possible anaesthesia of lip and tongue.

G. R. Ogden, Professor of Oral and Maxillofacial Surgery University of Dundee Dental Hospital and School

1. Savin J, Ogden G R. Third molar surgery-a preliminary report on aspects affecting quality of life in the early post-operative period. Br J Oral Maxillofac Surg 1997; 35: 246-253.

2 Ruta D A, Bissias E, Ogston S, Ogden G R. Assessing health outcomes after extraction of third molars: post-operative symptom severity (PoSSe) scale. Br J Oral Maxillofac Surg 2000; 38: 480-487. 